\title{
Association between use of disease-modifying antirheumatic drugs and diabetes in patients with ankylosing spondylitis, rheumatoid arthritis, or psoriasis/psoriatic arthritis: a nationwide, population-based cohort study of 84,989 patients
}

This article was published in the following Dove Press journal:

Therapeutics and Clinical Risk Management

2 May 2017

Number of times this article has been viewed

\section{Hsin-Hua Chen ${ }^{1-7}$ \\ Der-Yuan Chen ${ }^{1-6}$ \\ Chi-Chen Lin ${ }^{1,2}$ \\ Yi-Ming Chen ${ }^{1-4}$ \\ Kuo-Lung Lai ${ }^{3,4}$ \\ Ching-Heng Lin'}

'Department of Medical Research, Taichung Veterans General Hospital, ${ }^{2}$ Institute of Biomedical Science and Rong Hsing Research Center for Translational Medicine, ChungHsing University, Taichung, ${ }^{3} \mathrm{~S} c h o o l$ of Medicine, National Yang-Ming University, Taipei, ${ }^{4}$ Division of Allergy, Immunology and Rheumatology, Department of Internal Medicine, Taichung Veterans General Hospital, ${ }^{5}$ School of Medicine, Chung-Shan Medical University, ${ }^{6}$ Department of Medical Education, Taichung Veterans General Hospital, Taichung, ${ }^{7}$ Institute of Public Health and Community Medicine Research Center, National Yang-Ming University, Taipei, Taiwan

Correspondence: Hsin-Hua Chen Division of Allergy, Immunology and Rheumatology, Department of Internal Medicine, Taichung Veterans General Hospital, No 1650, Section 4, Taiwan Boulevard, Taichung 40705, Taiwan Tel +886 423592525 (ext 4300)

Fax +886 423503285

Email shc5555@hotmail.com
Purpose: The aim of this study is to investigate the association between the use of diseasemodifying antirheumatic drugs (DMARDs) and diabetes mellitus (DM) in patients with ankylosing spondylitis (AS), rheumatoid arthritis (RA), or psoriasis/psoriatic arthritis (PS/PSA).

Patients and methods: This retrospective cohort study used a nationwide, population-based administrative database to enroll 84,989 cases with AS, RA, or PS/PSA who initiated treatment with anti-tumor necrosis factor (anti-TNF) drugs or nonbiologic DMARDs. Multivariable analysis was used to estimate the effect of different therapies on the risk of DM.

Results: The incidence rates of DM per 1,000 person-years were 8.3 for users of anti-TNF drugs, 13.3 for users of cyclosporine (CSA), 8.4 for users of hydroxychloroquine (HCQ), and 8.1 for users of other nonbiologic DMARDs. Compared with the users of nonbiologic DMARDs, the multivariate-adjusted hazard ratios (aHRs) for DM were significantly lower for those who used anti-TNF drugs with HCQ (aHR: 0.49, 95\% confidence interval [CI]: 0.36-0.66) and those who used HCQ alone (aHR: 0.70, 95\% CI: $0.63-0.78$ ), but not for those who used anti-TNFs without HCQ (aHR: 1.23, 95\% CI: 0.94-1.60) or CSA (aHR: 1.14, 95\% CI: 0.77-1.70).

Conclusion: The aHR for DM was lowest for patients with RA and PS/PSA who initiated treatment with an anti-TNF agent with concomitant HCQ, followed by HCQ users. Those who used anti-TNF agents without HCQ and other nonbiologic DMARDs had a similar risk of DM.

Keywords: ankylosing spondylitis, rheumatoid arthritis, psoriasis, psoriatic arthritis, diabetes mellitus

\section{Introduction}

Individuals with chronic inflammation have an increased risk of cardiovascular disease, ${ }^{1-3}$ and some of this increased risk is mediated by the development of increased insulin resistance and diabetes mellitus (DM). ${ }^{4,5}$ More specifically, cardiovascular disease is a major cause of death in patients with rheumatoid arthritis (RA), ${ }^{6}$ and individuals with RA or psoriasis (PS) have an increased risk of insulin resistance and DM. ${ }^{7}$ Other researches have indicated that patients with PS may experience accelerated development of insulin resistance and onset of DM. ${ }^{8,9}$ Patients with systemic lupus erythematosus (SLE) also have an increased risk of DM. ${ }^{10}$

The tumor necrosis factor (TNF) is a cytokine that performs diverse functions, and its actions may underlie the relationship of autoimmune diseases with an increased 
insulin resistance and DM. ${ }^{11,12}$ TNF inhibitors, such as etanercept (ETN) and adalimumab (ADA), may be prescribed to patients with RA, PS and psoriatic arthritis (PSA), and other autoimmune diseases. Thus, it seems possible that patients given an anti-TNF drug for treatment of an autoimmune disease may also benefit by having delayed development of DM and reduced risk of cardiovascular disease. In fact, a recent study of RA patients reported that patients who used a TNF inhibitor had a reduced risk of DM. ${ }^{13}$ These results are in line with the in vitro study by Zhou et al, ${ }^{14}$ who studied cultured human adipocytes and concluded that TNF increases the sensitivity of adipocytes to insulin.

Hydroxychloroquine (HCQ) is best known as a traditional treatment for malaria, but is also indicated for patients with RA and SLE. HCQ may also inhibit the development of insulin resistance and DM in patients with RA and PS. ${ }^{15-17}$ The mechanism of action of HCQ in the prevention of insulin resistance and DM is not completely resolved, but may involve blocking of toll-like receptors 7 and 9 and inhibition of the production of interferon- $\alpha{ }^{18}$

To the best of our knowledge, no study has yet examined the association between the use of a disease-modifying antirheumatic drug (DMARD) and the risk of DM in a nationwide cohort of patients with ankylosing spondylitis (AS), RA, and PS/PSA. This study used the Taiwanese National Health Insurance Research Database (NHIRD) to estimate the risk of incident DM in a nationwide cohort of patients who initiated treatment with an anti-TNF agent, cyclosporine (CSA), HCQ, or another nonbiologic DMARD for RA, PS/PSA, or AS.

\section{Patients and methods}

\section{Study design, data source, and patient selection}

This was a retrospective cohort study that used data from the National Health Insurance (NHI) program of Taiwan, which covers more than $99 \%$ of the population. The National Health Research Institute (NHRI) manages the NHIRD and releases claims data of ambulatory care, inpatient services, dental services, traditional Chinese medical services, and prescription drug use for research purposes. All data were encrypted to ensure anonymity before being sent to the NHRI, and data that may enable identification of individuals were removed before release to researchers. Some individual information, such as use of tobacco and alcohol, examination data, and family history of DM, are not included in the NHIRD. To improve the accuracy of coding, the Bureau of NHI routinely performs random checks of patient charts to ensure coding validity. ${ }^{19}$
The present study utilized ambulatory, inpatient, and enrollment claims data from the NHIRD for the period 1999-2011 to identify all the patients, regardless of age, with diagnoses of AS (at least 3 outpatient department [OPD] visits with a diagnosis based on the International Classification of Diseases-9 [ICD9] code 720.0), RA (at least 3 OPD visits with a diagnosis of ICD9 code 714.0 and with a catastrophic illness certificate), or PS/PSA (at least 3 OPD visits with a diagnosis of code ICD9 696.0-1). All enrolled patients were given prescriptions for an anti-TNF agent (ETN or ADA) or a traditional synthetic DMARD (oral CSA, oral or injectable methotrexate [MTX], HCQ, sulfasalazine [SSZ], leflunomide [LEF], or azathioprine [AZA]). Although HCQ is not in the treatment guidelines for these autoimmune diseases, some clinical practitioners in Taiwan continue to prescribe HCQ for these patients, as an off-label treatment. CSA is sometimes used with MTX for the treatment of RA, and we examined this drug because studies of transplant patients indicate that it may increase the risk of DM. In Taiwan, patients with some catastrophic illnesses including RA receive catastrophic illness certificates after a thorough review of clinical data by at least 2 specialists; this study used the NHI catastrophic illness files, including ambulatory, inpatient, and enrollment claims, to identify incident RA cases. Patients with diagnoses of DM (ICD9-CM code 250.X) before initiation of DMARD therapy were excluded. This research was approved by the Institutional Review Board (IRB) I \& II of Taichung Veterans General Hospital (IRB number: CE16217B). Informed consent was not obtained because all personal details traced had been anonymized before analysis.

\section{DMARD use in Taiwan}

Based on a review of the NHIRD, the commonly used drugs (in the order of frequency) for AS are nonsteroidal antiinflammatory drugs (NSAIDs), salazopyrine, corticosteroid, anti-TNF agent, and HCQ; those used for RA are NSAIDs, corticosteroid, MTX, salazopyrine, LEF, anti-TNF agent, CSA, and AZA; those used for PS are MTX, CSA, LEF, salazopyrine, and anti-TNF agent; and those used for PSA are NSAIDs, corticosteroid, MTX, LEF, salazopyrine, and anti-TNF agent. Anti-TNF therapy is currently a second-line treatment in Taiwan. The National Health Insurance Administration (NHIA), which manages the NHI program, approved the use of ETN for RA treatment in 2003, and ETN and ADA for the treatment of AS and PS/PSA in 2009. Certified rheumatologists can initiate anti-TNF therapy for patients with inadequate response or intolerant to conventional standard 
treatment according to the NHIA guideline,${ }^{20}$ which is based on the National Institute for Health and Care Excellence guideline $^{21}$ but with slight modification.

\section{Index dates}

The drug index date was defined as the first date of observation of AS, RA, or PS/PSA from an ambulatory visit at which a prescription for an anti-TNF agent or a traditional synthetic DMARD was given. The DM index date was the date on which there was a diagnosis of DM (ICD9-CM code 250.X), which includes type-1 and type-2 DM, and concurrent prescription of an anti-diabetes medication for more than 28 days after January 1, 2001.

\section{Outcome variables and potential confounders}

The outcome variable was the time from the drug index date to the first date of an ambulatory visit with a diagnosis of DM (ICD9-CM code 250.X) and the concurrent prescription of any anti-diabetes drugs. The censored date was the last date of prescription of a DMARD. The potential confounders considered were age, sex, disease duration, Charlson comorbidity index (CCI), and prior or concomitant use of a corticosteroid.

\section{Statistical analysis}

For comparison of the effect of different treatments on DM, patients were classified into 4 groups according to DMARD use; a fifth group was used for Kaplan-Meier survival analysis. Comparison of continuous variables employed an analysis of variance (ANOVA) test, and comparison of categorical variables employed a Chi-square test. The effect of different DMARDs on risk of DM was determined by calculating adjusted hazard ratios (aHRs) and 95\% confidence intervals (CIs) using Cox proportional regression analysis after adjustment for potential confounders. A 2-tailed $P$-value less than 0.05 was considered statistically significant. Age is presented as mean and standard deviation, and 1-way ANOVA with a Bonfferroni post hoc test was used to evaluate differences among the 4 groups. Categorical data are presented as number and percentage, and the 4 groups were compared with Fisher's exact test. The cumulative incidence of DM was calculated as 1 minus the Kaplan-Meier estimate of DM-free rate, and the cumulative incidence of DM in the 4 or 5 medication groups (with HCQ as the fifth group) was determined with the log-rank test. All statistical analyses were conducted using SPSS version 18.0 for Windows (SPSS Inc., Chicago, IL, USA).

\section{Results}

This retrospective cohort study used a nationwide, populationbased administrative database (1999-2011) to enroll 84,989 patients. There were 36,329 patients with AS, 33,112 patients with RA, and 15,638 patients with PS/PSA. All enrolled patients initiated treatment with an anti-TNF agent or a nonbiologic DMARD. We categorized patients into 4 mutually exclusive groups based on DMARD use: 1) anti-TNF agent with or without another DMARD $(n=6,824)$; 2$)$ CSA without an anti-TNF agent or HCQ $(n=1,084)$; 3) HCQ without an anti-TNF agent or CSA $(n=27,033)$; and 4) other nonbiologic DMARD without an anti-TNF agent, CSA, or HCQ ( $n=50,048$; reference group).

\section{Characteristics of enrolled patients}

Table 1 summarizes the characteristics of patients in these 4 groups. Patients in the reference group (mean age: 39.0 years) were significantly younger than those in the other groups (anti-TNF: 49.2 years, CSA: 48.0 years, HCQ: 50.5 years). Most patients in the anti-TNF, CSA, and HCQ groups were female $(66.7 \%-72.6 \%)$, but most of the patients in the reference group were male (67.3\%). Most patients in the anti-TNF, CSA, and HCQ groups had RA $(72.9 \%-78.9 \%)$, but most patients in the reference group had AS (61.8\%) or PS/PSA (26.0\%). Most subjects in the CSA, HCQ, and reference groups had a disease duration less than 5 years $(77.2 \%-88.9 \%)$, but only $38.2 \%$ of subjects in the anti-TNF group had a disease duration less than 5 years. Most patients in the anti-TNF and CSA groups had pretreatment CCI scores of 1-4 (79.5\% and 77.3\%, respectively), most patients in the HCQ group had CCI scores of 0-1 (76.6\%), and most patients in the reference group had CCI scores of $0(73.8 \%)$. Analysis of the pretreatment use of prednisoloneequivalents within 1 year indicated that most patients in the anti-TNF group (69.7\%) and CSA group (64.2\%) took more than $1 \mathrm{mg} /$ day, and most patients in the HCQ group (80.1\%) and reference group (92.2\%) took no more than $1 \mathrm{mg} /$ day. There were significantly fewer overall patients in the reference group who used MTX ( $=13,893,31.1 \%, P<0.001)$, but significantly more patients in the reference group with AS who used MTX (n=3,159, 22.7\%, P<0.001).

\section{Incidence of DM in patients who used different DMARDs}

Table 2 shows the incidence of newly diagnosed DM in each of the 4 groups. There were 127 cases of DM in the antiTNF group, 26 in the CSA group, 1,000 in the HCQ group, and 1,486 in the reference group. The CSA group had the 
Table I Characteristics of Taiwanese patients with AS, RA, or PS/PSA who initiated therapy with a DMARD from I999 to 20 I I

\begin{tabular}{|c|c|c|c|c|c|}
\hline \multirow[t]{2}{*}{ Variable } & \multicolumn{4}{|c|}{ DMARD therapy } & \multirow[t]{2}{*}{$P$-value } \\
\hline & $\begin{array}{l}\text { Anti-TNF } \\
(n=6,824)\end{array}$ & $\begin{array}{l}\text { CSA }^{a} \\
(n=2,940)\end{array}$ & $\begin{array}{l}\mathrm{HCQ}^{\mathrm{a}} \\
(\mathrm{n}=\mathbf{2 7}, \mathbf{7 8 3})\end{array}$ & $\begin{array}{l}\text { Reference }^{a} \\
(n=44,635)\end{array}$ & \\
\hline Age at initiation of DMARDs (years) & $49.2(14.9)$ & $43.6(15.7)$ & $50.3(\mid 5.5)$ & $38.2(14.7)$ & $<0.001$ \\
\hline \multicolumn{6}{|l|}{ Sex } \\
\hline Female & $4,553(66.7 \%)$ & I,3। 3 (44.7\%) & $20,058(72.2 \%)$ & $13,200(29.6 \%)$ & \multirow[t]{2}{*}{$<0.001$} \\
\hline Male & 2,27 I $(33.3 \%)$ & I,627 (55.3\%) & $7,725(27.8 \%)$ & 31,435 (70.4\%) & \\
\hline \multicolumn{6}{|l|}{ Disease group } \\
\hline AS & $1,126(16.5 \%)$ & $204(6.9 \%)$ & $4,384(15.8 \%)$ & $30,473(68.3 \%)$ & \multirow[t]{3}{*}{$<0.001$} \\
\hline RA & 4,975 (72.9\%) & $874(29.7 \%)$ & $21,530(77.5 \%)$ & $3,148(7.1 \%)$ & \\
\hline PS/PSA & $723(10.6 \%)$ & I,862 (63.3\%) & $1,869(6.7 \%)$ & II,0।4 (24.7\%) & \\
\hline \multicolumn{6}{|l|}{ Disease duration } \\
\hline$\leq 5$ years & $2,606(38.2 \%)$ & I,756 (59.7\%) & $24,735(89.0 \%)$ & $35,343(79.2 \%)$ & \multirow[t]{3}{*}{$<0.001$} \\
\hline$>5-10$ years & $2,593(38.0 \%)$ & $788(26.8 \%)$ & $2,396(8.6 \%)$ & $6,033(13.5 \%)$ & \\
\hline$>10$ years & $1,625(23.8 \%)$ & $396(13.5 \%)$ & $652(2.3 \%)$ & $3,259(7.3 \%)$ & \\
\hline \multicolumn{6}{|l|}{ Pretreatment $\mathrm{CCl}$ score } \\
\hline 0 & 1,131 (16.6\%) & I,6I8 (55.0\%) & $10,525(37.9 \%)$ & $33,628(75.3 \%)$ & \multirow[t]{4}{*}{$<0.001$} \\
\hline 1 & $3,428(50.2 \%)$ & $734(25.0 \%)$ & $10,822(39.0 \%)$ & $7,082(15.9 \%)$ & \\
\hline $2-4$ & I,998 (29.3\%) & $460(15.6 \%)$ & 5,467 (19.7\%) & $3,015(6.8 \%)$ & \\
\hline$\geq 5$ & $267(3.9 \%)$ & $128(4.4 \%)$ & 969 (3.5\%) & $910(2.0 \%)$ & \\
\hline \multicolumn{6}{|l|}{ Pretreatment prednisolone dose ${ }^{b}$} \\
\hline None & $1,536(22.5 \%)$ & $\mathrm{I}, 245$ (42.3\%) & $10,098(36.3 \%)$ & $30,631(68.6 \%)$ & \multirow[t]{3}{*}{$<0.001$} \\
\hline$\leq \mathrm{I} \mathrm{mg} /$ day & $535(7.8 \%)$ & $785(26.7 \%)$ & $12,279(44.2 \%)$ & $10,592(23.7 \%)$ & \\
\hline$>1 \mathrm{mg} /$ day & $4,753(69.7 \%)$ & $910(31.0 \%)$ & $5,406(19.5 \%)$ & $3,4 \mid 2(7.6 \%)$ & \\
\hline \multicolumn{6}{|l|}{ MTX used } \\
\hline Total & $4,884(71.6 \%)$ & $2,1111(71.8 \%)$ & I5,029 (54.1\%) & 13,893 (31.1\%) & $<0.001$ \\
\hline AS & $212(4.3 \%)$ & $55(2.6 \%)$ & $890(5.9 \%)$ & $3,159(22.7 \%)$ & \multirow[t]{3}{*}{$<0.001$} \\
\hline RA & $4,272(87.5 \%)$ & $560(26.5 \%)$ & 13,440 (89.4\%) & I,945 (I4.0\%) & \\
\hline PS/PSA & $400(8.2 \%)$ & I,496 (70.9\%) & $699(4.7 \%)$ & $8,789(63.3 \%)$ & \\
\hline
\end{tabular}

Notes: 'Based on the DMARD medication use, the patients were categorized into 4 mutually exclusive groups: (I) anti-TNF: TNF inhibitors with or without other DMARDs; (2) CSA: without TNF inhibitors or HCQ; (3) HCQ: without TNF inhibitors or CSA; (4) reference: nonbiologic DMARDs without TNF inhibitors, CSA, or HCQ. ${ }^{\circ}$ Daily average-equivalent dose in the I year before the index date.

Abbreviations: AS, ankylosing spondylitis; RA, rheumatoid arthritis; PS/PSA, psoriasis/psoriatic arthritis; DMARD, disease-modifying antirheumatic drug; TNF, tumor necrosis factor; CSA, cyclosporine; HCQ, hydroxychloroquine; CCl, Charlson comorbidity index; MTX, methotrexate.

highest DM incident density (13.26 per 1,000 person-years), followed by the HCQ group (8.37 per 1,000 person years), the anti-TNF group ( 8.26 per 1,000 person-years), and the reference group (8.12 per 1,000 person-years).

Figure 1A shows the cumulative incidence of DM in each of these 4 groups. These results show that the anti-TNF group had a significantly lower cumulative incidence of DM than the CSA group $(P=0.014)$. The HCQ and reference groups also had significantly lower cumulative incidences of DM than the CSA group ( $P=0.022$ and $P=0.013$, respectively).

We further categorized anti-TNF users as those who did and did not use HCQ (Figure 1B). This analysis showed that there was no protective effect of using an anti-TNF agent when HCQ was not used concomitantly. In particular, the cumulative DM incidence in the group that used anti-TNF without HCQ was comparable to that of the CSA group, and

Table 2 Incidence of DM in patients who used different DMARDs

\begin{tabular}{lllll}
\hline & DMARD therapy & & & \\
\cline { 2 - 5 } & $\begin{array}{l}\text { Anti-TNF } \\
(\mathbf{n}=\mathbf{6 , 8 2 4})\end{array}$ & $\begin{array}{l}\mathbf{C S A}^{\mathbf{a}} \\
(\mathbf{n = 2 , 9 4 0 )}\end{array}$ & $\begin{array}{l}\mathbf{H C Q}^{\mathbf{a}} \\
(\mathbf{n}=\mathbf{2 7}, \mathbf{7 8 3})\end{array}$ & $\begin{array}{l}\text { Reference }^{\mathbf{a}} \\
(\mathbf{n}=\mathbf{4 4 , 6 3 5 )}\end{array}$ \\
\hline Sum of person-years & $43,964.5$ & $13,258.9$ & $39,458.5$ & $105,835.9$ \\
New diagnoses of DM & 127.0 & 138.0 & $1,073.0$ & $1,137.0$ \\
Incidence (per I,000 person-years) & 2.9 & 10.4 & 27.2 & 10.7 \\
\hline
\end{tabular}

Notes: 'Based on the DMARD medication use, the patients were categorized into 4 mutually exclusive groups: (I) anti-TNF: TNF inhibitors with or without other DMARDs; (2) CSA without TNF inhibitors or HCQ; (3) HCQ without TNF inhibitors or CSA; (4) reference: other nonbiologic DMARDs without TNF inhibitors, CSA, or HCQ. Abbreviations: DM, diabetes mellitus; DMARD, disease-modifying antirheumatic drug; TNF, tumor necrosis factor; CSA, cyclosporine; HCQ, hydroxychloroquine. 
A

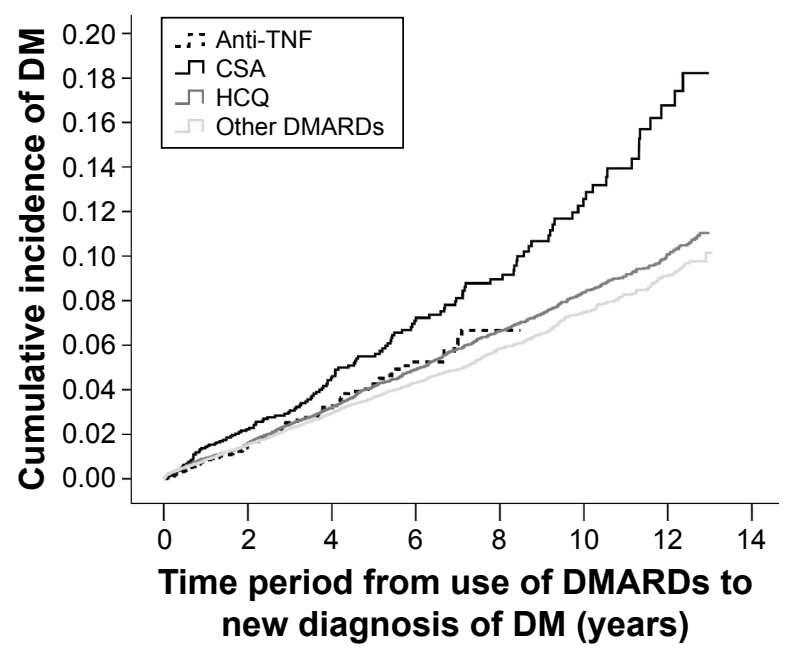

B

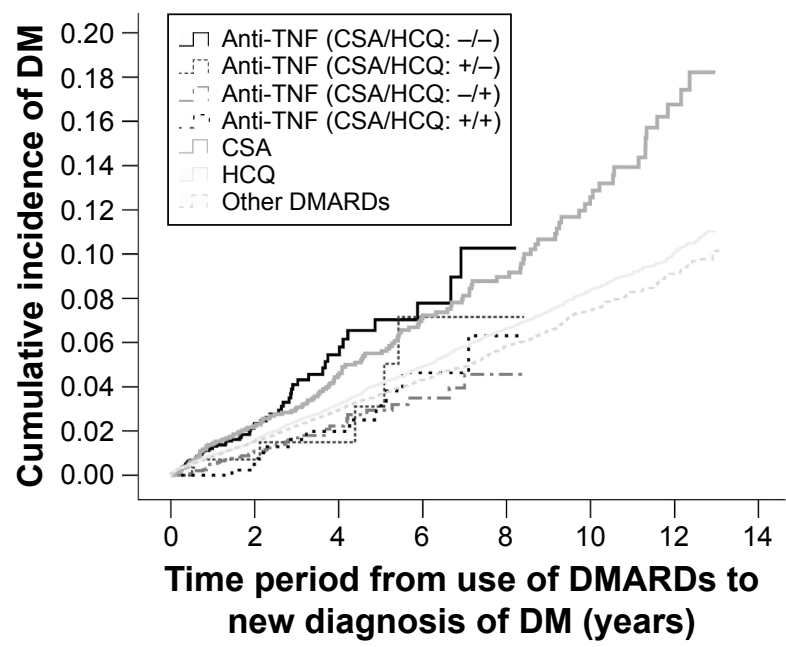

Figure I Cumulative incidence of DM with patients classified into 4 DMARD groups (A) and 5 DMARD groups (B). (A) The cumulative incidence of DM in the CSA group was significantly higher than that in the other 3 groups (vs anti-TNF: $P=0.014$; vs $\mathrm{HCQ}: P=0.022$; vs other nonbiologic $D M A R D s: ~ P=0.013$ ). (B) The cumulative incidence of DM was similar in the CSA group and the group that used anti-TNF without HCQ, and significantly lower in the other 3 groups (anti-TNF + HCQ, HCQ, other DMARDs); the cumulative incidence of DM in the anti-TNF + HCQ group was significantly lower than in the other 4 groups (vs anti-TNF without $\mathrm{HCQ}$ : $P<0.00 \mathrm{I}$; vs $C S A$ : $P<0.00 \mathrm{I}$; vs HCQ: $P=0.015$; vs other DMARDs: $P=0.031$ ).

Abbreviations: DM, diabetes mellitus; DMARD, disease-modifying antirheumatic drug; CSA, cyclosporine; TNF, tumor necrosis factor; HCQ, hydroxychloroquine.

significantly higher than the other 3 groups ( $P \leq 0.001$ for all comparisons). The protective effect of using an anti-TNF agent remained significant in the anti-TNF + HCQ group, and this group had a lower cumulative incidence of DM than all other groups (vs anti-TNF without HCQ: $P<0.001$; vs CSA: $P<0.001$; vs HCQ: $P=0.015$; vs reference group: $P=0.031)$.

\section{Risk factors for DM}

Table 3 summarizes the risk factors for DM in our population. The univariate analysis indicated that the following factors were associated with an increased risk of DM: advanced age, PA/PSA and RA rather than AS, longer disease duration ( $>5-10$ years vs $\leq 5$ years), higher pretreatment CCI score, and pretreatment use of prednisolone. In addition, relative to the use of reference therapy, use of an anti-TNF agent with HCQ had a significant protective effect, whereas the use of an anti-TNF agent alone and use of CSA increased the risk of DM.

Table 3 also shows the results of the multivariable analysis of the risk of DM. These results show that the following factors were independently associated with an increased risk of DM: advanced age (aHR: 1.04, $P<0.001$ ), PS/PSA rather than AS (aHR: $2.44, P<0.001$ ), pretreatment CCI score of $2-4$ (aHR: $1.29, P<0.001$ ) or 5 or more (aHR: 1.38, $P=0.006$ ) rather than CCI score of 0 , and pretreatment use of prednisolone at $1 \mathrm{mg}$ /day or less (aHR: 1.26, $P<0.001$ ) or more than $1 \mathrm{mg} /$ day (aHR: $1.30, P<0.001$ ) relative to no prednisolone use. These results also show that relative to the use of the reference therapy, the use of an anti-TNF agent with HCQ (aHR: 0.49, $P<0.001$ ) and use of HCQ alone (aHR: $0.70, P<0.001$ ) had protective effects. In addition, the use of prednisolone, MTX, SSZ, LEF, and AZA also protected against the development of DM.

\section{Risk factors for DM with stratification by disease duration}

We further analyzed the risk of DM with stratification by disease duration (Table 4). The multivariable Cox regression models showed that among those with disease durations of 5 years or less and 5-10 years, there were significant associations of advanced age, PS/PSA, and higher pretreatment CCI score with DM. The association of pretreatment prednisolone use with DM occurred in those with a disease duration of 5 years or less, but not in those with disease durations of 5-10 years and more than 10 years. Relative to the reference treatment, anti-TNF + HCQ therapy was protective in those with disease durations of 5 years or less (aHR: $0.56, P=0.003$ ) and 5-10 years (aHR: 0.33, $P<0.001$ ); HCQ therapy had a protective effect in those with disease durations of 5 years or less (aHR: $0.71, P<0.001$ ), 5-10 years (aHR: 0.61, $P=0.017$ ), and more than 10 years (aHR: 0.21, $P=0.040$ ). Prednisolone, MTX, SSZ, and LEF were protective for those with a disease duration of 5 or fewer years, but provided decreasing benefit for those with disease for more than 5 years. 
Table 3 Univariate (crude HR) and multivariate (adjusted HR) analysis of factors associated with DM

\begin{tabular}{|c|c|c|c|c|}
\hline Variable & Crude HR $(95 \% \mathrm{Cl})$ & $P$-value & Adjusted HR (95\% Cl) & $P$-value \\
\hline Age & $1.04(1.04,1.04)$ & $<0.00 I^{*}$ & $1.03(1.03,1.04)$ & $<0.00 I^{*}$ \\
\hline Sex (male to female) & $0.90(0.83,0.97)$ & $0.008^{*}$ & $1.05(0.96,1.15)$ & 0.299 \\
\hline \multicolumn{5}{|l|}{ Disease group } \\
\hline AS & Reference & & Reference & \\
\hline RA & $1.99(1.81,2.20)$ & $<0.00 I^{*}$ & $1.90(1.61,2.25)$ & $<0.00 I^{*}$ \\
\hline PS/PSA & $3.50(3.13,3.91)$ & $<0.001 *$ & $3.09(2.63,3.62)$ & $<0.00 I^{*}$ \\
\hline \multicolumn{5}{|l|}{ Disease duration } \\
\hline$\leq 5$ years & Reference & & Reference & \\
\hline$>5-10$ years & $1.45(1.28,1.64)$ & $<0.001 *$ & $0.82(0.71,0.94)$ & $0.004 *$ \\
\hline$>10$ years & $1.22(0.92,1.63)$ & 0.168 & $0.5 I(0.38,0.69)$ & $<0.00 I^{*}$ \\
\hline \multicolumn{5}{|l|}{ Pretreatment $\mathrm{CCl}$} \\
\hline 0 & Reference & & Reference & \\
\hline 1 & $1.15(1.04,1.26)$ & $0.005^{*}$ & $1.04(0.94,1.15)$ & 0.459 \\
\hline $2-4$ & $1.87(1.68,2.08)$ & $<0.00 I^{*}$ & $1.28(1.13,1.44)$ & $<0.00 I^{*}$ \\
\hline$\geq 5$ & $1.96(1.56,2.47)$ & $<0.00 I^{*}$ & $1.36(1.08,1.72)$ & $0.010^{*}$ \\
\hline \multicolumn{5}{|l|}{ Pretreatment prednisolone dose $\mathrm{e}^{\mathrm{a}}$} \\
\hline None & Reference & & Reference & \\
\hline$\leq \mathrm{I} \mathrm{mg} /$ day & $1.36(1.25,1.48)$ & $<0.00 I^{*}$ & $1.35(1.22,1.48)$ & $<0.00 I^{*}$ \\
\hline$>1 \mathrm{mg} /$ day & $1.47(1.32,1.64)$ & $<0.00 I^{*}$ & $\mathrm{I} .45(1.27, \mathrm{I} .65)$ & $<0.00 I^{*}$ \\
\hline DMARD group & Reference & & Reference & \\
\hline Anti-TNF (CSA/HCQ: -I-) & $1.64(1.29,2.10)$ & $<0.00 I^{*}$ & $1.18(0.89,1.56)$ & 0.244 \\
\hline Anti-TNF (CSA/HCQ: $+/-)$ & $0.98(0.44,2.18)$ & 0.957 & $0.72(0.32,1.62)$ & 0.428 \\
\hline Anti-TNF (CSA/HCQ: $-/+)$ & $0.73(0.54,1.01)$ & 0.056 & $0.44(0.31,0.63)$ & $<0.00 I^{*}$ \\
\hline Anti-TNF (CSA/HCQ: +/+) & $0.84(0.47,1.48)$ & 0.541 & $0.65(0.36,1.17)$ & 0.152 \\
\hline CSA & $1.66(1.39,1.98)$ & $<0.00 I^{*}$ & $0.90(0.75,1.08)$ & 0.256 \\
\hline $\mathrm{HCQ}$ & $1.12(1.03,1.21)$ & $0.011 *$ & $0.57(0.50,0.65)$ & $<0.00 I^{*}$ \\
\hline Prednisolone & $0.81(0.74,0.89)$ & $<0.00 I^{*}$ & $0.67(0.60,0.74)$ & $<0.00 I^{*}$ \\
\hline MTX & $0.95(0.88,1.03)$ & 0.230 & $0.58(0.53,0.64)$ & $<0.00 I^{*}$ \\
\hline SSZ & $0.42(0.38,0.45)$ & $<0.001 *$ & $0.65(0.59,0.7 I)$ & $<0.00 I^{*}$ \\
\hline LEF & $0.60(0.50,0.7 \mathrm{I})$ & $<0.00 I^{*}$ & $0.62(0.5 \mathrm{I}, 0.74)$ & $<0.001 *$ \\
\hline AZA & $0.99(0.83,1.18)$ & 0.929 & $0.96(0.8 \mathrm{I}, \mathrm{I} .15)$ & 0.694 \\
\hline
\end{tabular}

Notes: Reference for DMARD group: other nonbiologic DMARDs without TNF inhibitors, CSA, or HCQ. aDaily average-equivalent dose in the I year before the index date. $* P<0.05$ indicates a significant association with $D M$.

Abbreviations: HR, hazard ratio; DM, diabetes mellitus; Cl, confidence interval; AS, ankylosing spondylitis; RA, rheumatoid arthritis; PS/PSA, psoriasis/psoriatic arthritis; $\mathrm{CCl}$, Charlson comorbidity index; DMARD, disease-modifying antirheumatic drug; TNF, tumor necrosis factor; CSA, cyclosporine; HCQ, hydroxychloroquine; MTX, methotrexate; SSZ, sulfasalazine; LEF, leflunomide; AZA, azathioprine.

\section{Risk factors for DM with stratification by type of disease}

Finally, we analyzed the risk of DM with stratification by type of autoimmune disease (Table 5). This analysis showed significant associations of advanced age with DM in all 3 groups. In the AS group, males were more likely to develop DM than females (hazard ratio [HR]: 1.31, $P=0.012$ ). In the PS/PSA group, patients with a disease duration longer than 10 years were less likely to develop DM relative to those with a disease duration of 5 years or less (HR: $0.41, P<0.001$ ). In the AS and RA groups, patients with a high pretreatment CCI score and those who used prednisolone before treatment had an increased risk of DM. In the AS group, there were no newly diagnosed cases of DM among those given
anti-TNF + HCQ therapy, and the use of other drug regimens had no significant effect on the development of DM. In the RA group, anti-TNF + HCQ therapy (HR: $0.45, P<0.001$ ) and HCQ alone (HR: $0.66, P<0.001$ ) had significant protective effects. In the PS/PSA group, HCQ had a significant protective effect (HR: 0.66, $P=0.004$ ).

\section{Discussion}

This study is the first population-based cohort study to examine the association between the use of different DMARDs and DM among patients with AS, RA, or PS/PSA. Our major finding is that the risk of DM was lowest for patients with RA or PS/PSA who initiated treatment with an anti-TNF agent and concomitant HCQ, followed by those 
Table 4 Multivariable analysis of risk factors for DM with stratification by disease duration

\begin{tabular}{|c|c|c|c|c|c|c|}
\hline \multirow[t]{2}{*}{ Variable } & \multicolumn{2}{|l|}{$\begin{array}{l}\text { Duration } \leq 5 \text { years } \\
(n=64,440)\end{array}$} & \multicolumn{2}{|l|}{$\begin{array}{l}\text { Duration }>5-10 \text { years } \\
(n=|I, 8| 0)\end{array}$} & \multicolumn{2}{|l|}{$\begin{array}{l}\text { Duration }>10 \text { years } \\
(n=5,932)\end{array}$} \\
\hline & Adjusted HR (95\% Cl) & $P$-value & Adjusted HR (95\% Cl) & $P$-value & Adjusted HR (95\% Cl) & $P$-value \\
\hline Age & $1.04(1.03,1.04)$ & $<0.00 I^{*}$ & $1.03(1.02,1.04)$ & $<0.00 I^{*}$ & $1.02(1.00,1.04)$ & 0.109 \\
\hline Sex (male to female) & $1.07(0.97,1.18)$ & 0.196 & $0.96(0.73,1.24)$ & 0.732 & $0.91(0.47,1.73)$ & 0.763 \\
\hline \multicolumn{7}{|l|}{ Disease group } \\
\hline AS & Reference & & Reference & & Reference & \\
\hline RA & $1.82(1.52,2.18)$ & $<0.001 *$ & $2.79(1.51,5.17)$ & $0.001 *$ & $0.83(0.23,2.97)$ & 0.773 \\
\hline PS/PSA & $2.90(2.43,3.44)$ & $<0.00 I^{*}$ & $4.96(2.93,8.43)$ & $<0.00 I^{*}$ & $0.82(0.32,2.14)$ & 0.686 \\
\hline \multicolumn{7}{|l|}{ Pretreatment $\mathrm{CCl}$} \\
\hline 0 & Reference & & Reference & & Reference & \\
\hline 1 & $1.04(0.93,1.17)$ & 0.445 & $0.88(0.63,1.23)$ & 0.470 & $2.55(1.26,5.17)$ & $0.009 *$ \\
\hline $2-4$ & $\mathrm{I} .24(\mathrm{I} .09, \mathrm{I} .4 \mathrm{I})$ & $0.00 I^{*}$ & $1.39(0.99,1.95)$ & 0.060 & $2.26(0.96,5.34)$ & 0.063 \\
\hline$\geq 5$ & $1.41(1.09,1.82)$ & $0.009 *$ & $1.15(0.62,2.14)$ & 0.656 & $1.66(0.37,7.40)$ & 0.506 \\
\hline \multicolumn{7}{|l|}{ Pretreatment prednisolone dose $\mathrm{e}^{\mathrm{a}}$} \\
\hline None & Reference & & Reference & & Reference & \\
\hline$\leq \mathrm{I} \mathrm{mg} /$ day & $1.35(1.22,1.50)$ & $<0.00 I^{*}$ & $1.28(0.95,1.73)$ & 0.101 & I.48 $(0.73,2.99)$ & $0.28 \mathrm{I}$ \\
\hline$>1 \mathrm{mg} /$ day & $1.44(1.25,1.66)$ & $<0.00 I^{*}$ & $1.61(1.12,2.32)$ & $0.009 *$ & $1.21(0.52,2.80)$ & 0.658 \\
\hline DMARD group & Reference & & Reference & & Reference & \\
\hline Anti-TNF (CSA/HCQ: -/-) & $0.87(0.53, \mathrm{I} .4 \mathrm{I})$ & 0.568 & $1.10(0.64,1.90)$ & 0.719 & $0.68(0.30,1.55)$ & 0.356 \\
\hline Anti-TNF (CSA/HCQ: +/-) & $1.20(0.44,3.26)$ & 0.715 & $0.44(0.10,1.86)$ & 0.261 & NA & \\
\hline Anti-TNF (CSA/HCQ: -/+) & $0.4 \mathrm{I}(0.26,0.67)$ & $<0.00 I^{*}$ & $0.42(0.22,0.78)$ & $0.006 *$ & $0.20(0.04,1.11)$ & 0.066 \\
\hline Anti-TNF (CSA/HCQ: +/+) & I.I5 $(0.60,2.20)$ & 0.675 & $0.17(0.04,0.75)$ & $0.019 *$ & NA & 0.984 \\
\hline CSA & $0.92(0.75,1.14)$ & 0.440 & $0.79(0.52,1.21)$ & 0.277 & $0.31(0.07,1.34)$ & 0.117 \\
\hline HCQ & $0.57(0.50,0.66)$ & $<0.00 I^{*}$ & $0.5 \mathrm{I}(0.34,0.77)$ & $0.001 *$ & $0.12(0.03,0.56)$ & $0.007^{*}$ \\
\hline Prednisolone & $0.66(0.58,0.74)$ & $<0.00 I^{*}$ & $0.63(0.48,0.84)$ & $0.001 *$ & $1.33(0.69,2.59)$ & 0.394 \\
\hline MTX & $0.6 \mathrm{I}(0.55,0.67)$ & $<0.00 I^{*}$ & $0.47(0.35,0.63)$ & $<0.00 I^{*}$ & $0.34(0.17,0.67)$ & $0.002 *$ \\
\hline SSZ & $0.6 \mathrm{I}(0.55,0.67)$ & $<0.00 I^{*}$ & $0.97(0.75,1.24)$ & 0.792 & $0.57(0.29,1.10)$ & 0.094 \\
\hline LEF & $0.58(0.47,0.7 I)$ & $<0.00 I^{*}$ & $0.71(0.47,1.08)$ & 0.109 & $0.74(0.27,2.01)$ & 0.560 \\
\hline AZA & $1.01(0.83,1.22)$ & 0.934 & $0.65(0.36,1.19)$ & 0.162 & I.07 (0.3I, 3.7I) & 0.911 \\
\hline
\end{tabular}

Notes: Reference for DMARD group: other nonbiologic DMARDs without TNF inhibitors, CSA, or HCQ. NA indicates HR not available due to no new cases of DM. aDaily average-equivalent dose within I year before the index date. *Significant association with DM.

Abbreviations: DM, diabetes mellitus; HR, hazard ratio; Cl, confidence interval; AS, ankylosing spondylitis; RA, rheumatoid arthritis; PS/PSA, psoriasis/psoriatic arthritis; $\mathrm{CCl}$, Charlson comorbidity index; DMARD, disease-modifying antirheumatic drug; TNF, tumor necrosis factor; CSA, cyclosporine; HCQ, hydroxychloroquine; NA, not available; MTX, methotrexate; SSZ, sulfasalazine; LEF, leflunomide; AZA, azathioprine.

who used HCQ without an anti-TNF agent. Users of antiTNF agents without HCQ and users of other nonbiologic DMARDs had a similar risk of DM. Anti-TNF therapy and HCQ provided no benefit for patients with AS or PS/PSA. HCQ is rarely used for the treatment of PS/PSA because it may exacerbate the symptoms. ${ }^{22}$

The finding that anti-TNF therapy alone had no effect on the development of DM might seem surprising because a previous research has indicated that anti-TNF therapy reduced insulin resistance and the risk of DM in patients with RA. ${ }^{13}$ These apparently discrepant findings may be explained by the differences of ethnic groups, the criteria used for patient selection, and history of prednisolone usage. In addition, the protective effect of prednisolone on the development of DM is also unexpected because steroids may exacerbate DM.

A major strength of this study is that we used a nationwide population sample of Taiwanese men and women, most of whom are of Han Chinese ancestry. This design reduced selection bias, so we believe that the results are applicable to the general population of Taiwan. Another advantage of the present study relative to previous studies is that we adjusted for corticosteroid dosage, and did not simply classify patients as users and nonusers. Corticosteroids are indicated for patients with autoimmune diseases, especially PS and PSA, ${ }^{23}$ and it is well known that they can increase insulin resistance and promote the development of DM. ${ }^{24}$ Research that does not adjust for steroid dose may therefore overestimate the protective effect of anti-TNF agents because patients given anti-TNF agents may require lower doses of steroids. ${ }^{25}$

In agreement with our results, previous studies have also reported that HCQ can improve insulin tolerance or reduce the incidence of DM. ${ }^{26,27}$ Based on studies of an animal model, HCQ appears to directly affect the pancreas, leading to elevated blood insulin and reduced blood glucose. ${ }^{28}$ 
Table 5 Multivariable analysis of factors associated with DM with stratification by type of disease

\begin{tabular}{|c|c|c|c|c|c|c|}
\hline \multirow[t]{2}{*}{ Variable } & \multicolumn{2}{|l|}{ AS $(n=36,187)$} & \multicolumn{2}{|l|}{ RA $(n=30,527)$} & \multicolumn{2}{|l|}{ PS/PSA $(n=15,468)$} \\
\hline & Adjusted HR (95\% Cl) & $P$-value & Adjusted HR (95\% Cl) & $P$-value & Adjusted HR (95\% Cl) & $P$-value \\
\hline Age & $1.06(1.05,1.06)$ & $<0.00 I^{*}$ & $1.03(1.03,1.03)$ & $<0.00 I^{*}$ & $1.03(1.02,1.03)$ & $<0.00$ I* $^{*}$ \\
\hline Sex (male to female) & $1.33(1.07,1.64)$ & $0.009 *$ & $1.04(0.91,1.19)$ & $0.58 \mathrm{I}$ & $0.99(0.84,1.17)$ & 0.911 \\
\hline \multicolumn{7}{|l|}{ Disease duration } \\
\hline$\leq 5$ years & Reference & & Reference & & Reference & \\
\hline$>5-10$ years & $0.76(0.49,1.20)$ & 0.243 & $0.87(0.68,1.11)$ & 0.266 & $0.78(0.65,0.94)$ & $0.008^{*}$ \\
\hline$>10$ years & I.68 $(0.72,3.94)$ & 0.228 & $0.66(0.32,1.35)$ & 0.257 & $0.33(0.22,0.50)$ & $<0.00 I^{*}$ \\
\hline \multicolumn{7}{|l|}{ Pretreatment $\mathrm{CCl}$} \\
\hline 0 & Reference & & Reference & & Reference & \\
\hline I & $1.05(0.83,1.33)$ & 0.682 & $0.92(0.80,1.06)$ & 0.262 & $1.10(0.88,1.37)$ & 0.398 \\
\hline $2-4$ & $1.60(1.21,2.11)$ & $<0.001 *$ & $1.13(0.96,1.32)$ & 0.144 & $1.16(0.90,1.49)$ & 0.265 \\
\hline$\geq 5$ & $\mathrm{I} .48(0.88,2.5 \mathrm{I})$ & 0.141 & $1.19(0.87,1.64)$ & 0.280 & $1.30(0.82,2.06)$ & 0.260 \\
\hline \multicolumn{7}{|l|}{ Pretreatment prednisolone dose $\mathrm{e}^{\mathrm{a}}$} \\
\hline None & Reference & & Reference & & Reference & \\
\hline$\leq \mathrm{I} \mathrm{mg} /$ day & $1.35(1.11,1.64)$ & $0.003^{*}$ & $1.44(1.25,1.66)$ & $<0.001 *$ & $1.26(I .05, \mathrm{I} .5 \mathrm{I})$ & $0.012^{*}$ \\
\hline$>1 \mathrm{mg} /$ day & I.31 $(0.99,1.73)$ & 0.062 & $1.57(1.31,1.90)$ & $<0.00 I^{*}$ & $1.50(1.15,1.95)$ & $0.003 *$ \\
\hline DMARD group & Reference & & Reference & & Reference & \\
\hline Anti-TNF (CSA/HCQ: -I-) & $0.95(0.44,2.04)$ & 0.897 & $0.90(0.62,1.32)$ & 0.592 & $1.39(0.66,2.93)$ & 0.379 \\
\hline Anti-TNF (CSA/HCQ: +/-) & NA & & $0.83(0.36,1.92)$ & 0.666 & NA & \\
\hline Anti-TNF (CSA/HCQ: $-/+)$ & NA & & $0.39(0.27,0.58)$ & $<0.00 I^{*}$ & $0.44(0.06,3.16)$ & 0.412 \\
\hline Anti-TNF (CSA/HCQ: +/+) & NA & & $0.58(0.31,1.08)$ & 0.086 & NA & \\
\hline CSA & I.II $(0.40,3.06)$ & 0.844 & $0.92(0.60,1.43)$ & 0.719 & $0.8 \mathrm{I}(0.66, \mathrm{I} .0 \mathrm{I})$ & 0.059 \\
\hline $\mathrm{HCQ}$ & $0.54(0.39,0.75)$ & $<0.00 I^{*}$ & $0.5 \mathrm{I}(0.43,0.6 \mathrm{I})$ & $<0.00 I^{*}$ & $0.43(0.32,0.59)$ & $<0.00 I^{*}$ \\
\hline Prednisolone & $0.70(0.58,0.84)$ & $<0.001 *$ & $0.62(0.5 \mathrm{I}, 0.75)$ & $<0.00 I^{*}$ & $0.64(0.54,0.77)$ & $<0.00 I^{*}$ \\
\hline MTX & $0.83(0.66,1.05)$ & 0.127 & $0.59(0.52,0.67)$ & $<0.00 I^{*}$ & $0.35(0.28,0.43)$ & $<0.00 I^{*}$ \\
\hline SSZ & $0.35(0.25,0.47)$ & $<0.00 I^{*}$ & $0.69(0.61,0.78)$ & $<0.00 I^{*}$ & $0.63(0.53,0.76)$ & $<0.00 I^{*}$ \\
\hline LEF & $1.53(0.72,3.25)$ & 0.273 & $0.59(0.48,0.73)$ & $<0.001 *$ & $0.58(0.39,0.88)$ & $0.010^{*}$ \\
\hline AZA & $1.04(0.64,1.68)$ & 0.877 & $1.05(0.83,1.33)$ & 0.677 & $0.60(0.42,0.87)$ & $0.007^{*}$ \\
\hline
\end{tabular}

Notes: Reference for DMARD group: other nonbiologic DMARDs without TNF inhibitors, CSA, or HCQ. NA indicates HR not available due to no new cases of DM. aDaily average-equivalent dose within I year before the index date. *Significant association with DM.

Abbreviations: DM, diabetes mellitus; AS, ankylosing spondylitis; RA, rheumatoid arthritis; PS/PSA, psoriasis/psoriatic arthritis; HR, hazard ratio; Cl, confidence interval; $\mathrm{CCl}$, Charlson comorbidity index; DMARD, disease-modifying antirheumatic drug; TNF, tumor necrosis factor; CSA, cyclosporine; HCQ, hydroxychloroquine; NA, not available; MTX, methotrexate; SSZ, sulfasalazine; LEF, leflunomide; AZA, azathioprine.

Moreover, additional clinical research supports the use of HCQ for improving glucose metabolism in patients with rheumatic disease ${ }^{29}$ and for having other beneficial effects in patients with cancers, infections, and dyslipidemia. ${ }^{30}$

\section{Limitations}

Nonetheless, there were some limitations in this study. First, DM diagnoses were based on an administrative database, and therefore should have high accuracy. However, such non-differential misclassification is always biased toward the null, so this would not change the nature of the relationships we found, but only their significance. Second, the accuracy of diagnoses of AS and PS/PSA may be a concern, as is the diagnosis of nearly all autoimmune disorders. However, our RA diagnosis is not a concern because the certification of catastrophic illness requires validation by 2 or more rheumatologists and a comprehensive review of the patients' clinical data. Another limitation is that in examining the relationship of use of DMARDs with DM, the pathologies may differ for patients receiving different treatments. The lack of information on disease activity, functional disability, and structural damage means that we could not adjust for confounding by these factors in the statistical analysis. Finally, our administrative database does not provide laboratory or clinical information on disease severity. The disease duration was the time from date of diagnosis to date of initiating a DMARD. Therefore, those who initiated a DMARD earlier may have had a greater disease activity or severity. Disease severity may be related to the risk of DM, but was not available in the database.

\section{Conclusion}

We performed a nationwide survey to examine the association between the use of different DMARD therapies and the development of DM in patients diagnosed with AS, RA, or PS/PSA. The results indicated that the risk of DM was 
significantly lower in patients with RA or PS/PSA who initiated treatment with an anti-TNF agent with concomitant HCQ and was also lower in those who used HCQ but no anti-TNF agent. Users of anti-TNF agents without HCQ and users of other nonbiologic DMARDs had a similar risk of DM.

\section{Acknowledgments}

This study is based in part on data from the NHIRD provided by the NHIA, Ministry of Health and Welfare, and managed by the NHRIs (registered numbers 101095, 102148). The interpretation and conclusions contained herein do not represent those of the NHIA, Ministry of Health and Welfare, or NHRIs. The authors thank the Biostatistics Task Force of Taichung Veterans General Hospital, Taichung, Taiwan, for the statistical work. This study was supported in part by grants from Taichung Veterans General Hospital, Taiwan (TCVGH-1057314C). The abstract of this paper was presented at the EULAR 2016 conference as an oral presentation with interim findings. The oral presentation's abstract was published in "Oral Presentation Abstracts" in Annals of the Rheumatic Diseases journal (http://ard.bmj.com/content/75/ Suppl 2/119.3).

\section{Disclosure}

The authors declare that there are no conflicts of interest in this work.

\section{References}

1. Gabriel SE. Cardiovascular morbidity and mortality in rheumatoid arthritis. Am J Med. 2008;121(10 Suppl 1):S9-S14.

2. Solomon DH, Karlson EW, Rimm EB, et al. Cardiovascular morbidity and mortality in women diagnosed with rheumatoid arthritis. Circulation. 2003;107(9):1303-1307.

3. Gelfand JM, Neimann AL, Shin DB, Wang X, Margolis DJ, Troxel AB. Risk of myocardial infarction in patients with psoriasis. JAMA. 2006; 296(14):1735-1741.

4. Garg R, Tripathy D, Dandona P. Insulin resistance as a proinflammatory state: mechanisms, mediators, and therapeutic interventions. Curr Drug Targets. 2003;4(6):487-492.

5. Wellen KE, Hotamisligil GS. Inflammation, stress, and diabetes. J Clin Invest. 2005;115(5):1111-1119.

6. Wasko MC. Rheumatoid arthritis and cardiovascular disease. Curr Rheumatol Rep. 2008;10(5):390-397.

7. Chung CP, Oeser A, Solus JF, et al. Inflammation-associated insulin resistance: differential effects in rheumatoid arthritis and systemic lupus erythematosus define potential mechanisms. Arthritis Rheum. 2008; 58(7):2105-2112.

8. Qureshi AA, Choi HK, Setty AR, Curhan GC. Psoriasis and the risk of diabetes and hypertension: a prospective study of US female nurses. Arch Dermatol. 2009;145(4):379-382.

9. Solomon DH, Love TJ, Canning C, Schneeweiss S. Risk of diabetes among patients with rheumatoid arthritis, psoriatic arthritis and psoriasis. Ann Rheum Dis. 2010;69(12):2114-2117.

10. DREAM Trial Investigators; Bosch J, Yusuf S, Gerstein HC, et al. Effect of ramipril on the incidence of diabetes. $N$ Engl J Med. 2006;355(15): 1551-1562.
11. Van Doornum S, McColl G, Wicks IP. Accelerated atherosclerosis: an extraarticular feature of rheumatoid arthritis? Arthritis Rheum. 2002;46(4):862-873.

12. Dessein PH, Joffe BI, Stanwix AE. Inflammation, insulin resistance, and aberrant lipid metabolism as cardiovascular risk factors in rheumatoid arthritis. J Rheumatol. 2003;30(7):1403-1405.

13. Antohe JL, Bili A, Sartorius JA, et al. Diabetes mellitus risk in rheumatoid arthritis: reduced incidence with anti-tumor necrosis factor $\alpha$ therapy. Arthritis Care Res (Hoboken). 2012;64(2):215-221.

14. Zhou W, Yang P, Liu L, et al. Transmembrane tumor necrosis factoralpha sensitizes adipocytes to insulin. Mol Cell Endocrinol. 2015; 406:78-86.

15. Bili A, Sartorius JA, Kirchner HL, et al. Hydroxychloroquine use and decreased risk of diabetes in rheumatoid arthritis patients. J Clin Rheumatol. 2011;17(3):115-120.

16. Wasko MC, Hubert HB, Lingala VB, et al. Hydroxychloroquine and risk of diabetes in patients with rheumatoid arthritis. JAMA. 2007; 298(2):187-193.

17. Chen YM, Lin CH, Lan TH, et al. Hydroxychloroquine reduces risk of incident diabetes mellitus in lupus patients in a dose-dependent manner: a population-based cohort study. Rheumatology (Oxford). 2015; 54(7):1244-1249.

18. Costedoat-Chalumeau N, Dunogué B, Morel N, Le Guern V, GuettrotImbert G. Hydroxychloroquine: a multifaceted treatment in lupus. Presse Med. 2014;43(6 Pt 2):e167-e180.

19. Cheng TM. Taiwan's new national health insurance program: genesis and experience so far. Health Aff (Millwood). 2003;22(3):61-76.

20. Ministry of Health and Welfare. Part Six Supplementary Provisions, Article 83: the reimbursement restrictions of pharmaceuticals in the schedule are set forth in Attachment 6. The National Health Insurance Pharmaceutical Benefits and Reimbursement Schedule. 2013. Available from: http://law.moj.gov.tw/LawClass/LawSingleIf. aspx?Pcode $=$ L0060035\&FLNO=83. Accessed April 6, 2017.

21. National Institute for Health and Care Excellence. Rheumatoid arthritis in adults: management. 2009 [updated December 2015]. Available from: https://www.nice.org.uk/guidance/cg79

22. Gravani A, Gaitanis G, Zioga A, Bassukas ID. Synthetic antimalarial drugs and the triggering of psoriasis - do we need disease-specific guidelines for the management of patients with psoriasis at risk of malaria? Int J Dermatol. 2014;53(3):327-330.

23. Del Rosso J, Friedlander SF. Corticosteroids: options in the era of steroidsparing therapy. J Am Acad Dermatol. 2005;53(1 Suppl 1):S50-S58.

24. Greaves RF, Jevalikar G, Hewitt JK, Zacharin MR. A guide to understanding the steroid pathway: new insights and diagnostic implications. Clin Biochem. 2014;47(15):5-15.

25. Di Comite G, Rossi CM. Risk of diabetes in patients with rheumatoid arthritis taking hydroxychloroquine. JAMA. 2007;298(20):2368-2369; author reply 2369-2370.

26. Gonzalez-Gay MA, De Matias JM, Gonzalez-Juanatey C, et al. Anti-tumor necrosis factor-alpha blockade improves insulin resistance in patients with rheumatoid arthritis. Clin Exp Rheumatol. 2006;24(1):83-86.

27. Larsen CM, Faulenbach M, Vaag A, et al. Interleukin-1-receptor antagonist in type 2 diabetes mellitus. $N$ Engl $J$ Med. 2007;356(15): $1517-1526$.

28. Garcia-Webb P, Bonser AM. Insulin binding and degradation in isolated hepatocytes from streptozotocin injected rats. Biochem Biophys Res Commun. 1985;128(2):487-493.

29. Hage MP, Al-Badri MR, Azar ST. A favorable effect of hydroxychloroquine on glucose and lipid metabolism beyond its anti-inflammatory role. Ther Adv Endocrinol Metab. 2014;5(4):77-85.

30. Olsen NJ, Schleich MA, Karp DR. Multifaceted effects of hydroxychloroquine in human disease. Semin Arthritis Rheum. 2013;43(2): 264-272. 


\section{Publish your work in this journal}

Therapeutics and Clinical Risk Management is an international, peerreviewed journal of clinical therapeutics and risk management, focusing on concise rapid reporting of clinical studies in all therapeutic areas, outcomes, safety, and programs for the effective, safe, and sustained use of medicines. This journal is indexed on PubMed Central, CAS,

EMBase, Scopus and the Elsevier Bibliographic databases. The manuscript management system is completely online and includes a very quick and fair peer-review system, which is all easy to use. Visit http://www.dovepress.com/testimonials.php to read real quotes from published authors.

Submit your manuscript here: http://www.dovepress.com/therapeutics-and-clinical-risk-management-journal 